\title{
Aplicabilidade dos sistemas CAD/CAM em Prótese Total: revisão de literatura
}

\author{
Applicability of CAD/CAM systems to Total Prosthesis: literature review \\ Aplicabilidad de los sistemas CAD/CAM en Prótesis Total: revisión de literatura \\ Caroline Costa TAVARES ${ }^{1}$ \\ Julliana Cariry Palhano FREIRE ${ }^{2}$ \\ Stéphanie Cariry Palhano FREIRE ${ }^{3}$ \\ Eduardo DIAS-RIBEIRO ${ }^{4}$ \\ André Ulisses Dantas BATISTA ${ }^{5}$ \\ Especialista em Prótese Dentária, Faculdade COESP, João Pesso - PB, Brasil \\ ${ }^{2}$ Professora de Prótese Dentária da Universidade Estadual da Paraíba (UEPB) \\ Doutoranda em Clínica Odontológica pela Universidade Estadual da Paraíba, Campina Grande - PB, Brasil \\ ${ }^{3}$ Enfermeira. Hospital Universitário Alcides Carneiro, Campina Grande - PB, Brasil \\ ${ }^{4}$ Professor Doutor. Programa de Pós-Graduação em Odontologia, Universidade Federal da Paraíba (UFPB), João Pessoa - PB, Brasil \\ ${ }_{5}^{5}$ Professor Doutor. Programa de Pós-Graduação em Odontologia, Universidade Federal da Paraíba (UFPB), João Pessoa-PB, Brasil
}

\section{Resumo}

Introdução: A tecnologia $\mathrm{CAD} / \mathrm{CAM}$ possui múltiplas aplicações protéticas, incluindo a prótese total. Objetivos: realizar uma revisão de literatura sobre a aplicabilidade dos sistemas CAD/CAM na fabricação de próteses totais, a fim de elucidar as propriedades das próteses confeccionadas através dessa tecnologia e as características desse sistema. Metodologia: A busca por artigos científicos que contemplassem o tema foi realizada através das bases de dados PUBMED e SCIELO, sem restrição de período. Foi feita a análise dos títulos e resumos e em seguida o acesso ao texto completo. Resultados: Dentre as características do sistema CAD/CAM, os estudos citaram menor necessidade de consultas e tempo clínico. É preciso conhecimento da tecnologia pelo cirurgião-dentista e o maior custo laboratorial é uma das desvantagens de sua utilização. Quanto às propriedades, observa-se a redução da rugosidade e porosidade, assim como da adesão de Candida albicans à base da prótese e teor de monômero residual. Maior dureza de superfície é verificada em comparação com as próteses totais convencionais. Conclusão: Menor número de consultas e tempo clínico são características do uso do sistema CAD/CAM em prótese total. Maior dureza da prótese e redução da rugosidade, porosidade, adesão de $C$. albicans e do teor de monômero residual são propriedades que as próteses totais apresentam quando confeccionadas por essa tecnologia.

Descritores: Odontologia; Prótese Total; Projeto Auxiliado por Computador.

\section{Abstract}

Introduction: $\mathrm{CAD} / \mathrm{CAM}$ technology has multiple prosthetic applications, including complete denture. Objectives: To carry out a literature review on the applicability of CAD/CAM systems in the manufacture of complete dentures in order to elucidate the properties of denture made using this technology and the characteristics of this system. Methodology: The search for scientific articles that contemplated the theme was done through PUBMED and SCIELO databases, without period restriction. The titles and abstracts were analyzed and then access to the full text. Results: Among the characteristics of the CAD/CAM system, the studies cited a lower need for consultations and clinical time. It requires knowledge of the technology by the dental surgeon and the higher labor cost is one of the disadvantages of its use. As regards the properties, the reduction of the roughness and porosity, as well as the adhesion of Candida albicans to the base of the prosthesis and residual monomer content was observed. Higher hardness of surface is verified compared to conventional full dentures. Conclusion: Fewer appointments and clinical time are characteristics of the use of CAD/CAM system in complete denture. Higher hardness of the prosthesis and reduction of the roughness, porosity, adhesion of $C$. albicans and residual monomer content are properties that the complete dentures present when made by this technology.

Descriptors: Dentistry; Denture, Complete; Computer-Aided Design.

\section{Resumen}

Introducción: La tecnología CAD / CAM tiene múltiples aplicaciones protésicas, incluyendo la prótesis total. Objetivos: realizar una revisión de literatura sobre la aplicabilidad de los sistemas CAD / CAM en la fabricación de prótesis totales, a fin de elucidar las propiedades de las prótesis confeccionadas a través de esa tecnología y las características de ese sistema. Metodología: La búsqueda por artículos científicos que contemplaran el tema fue realizada a través de las bases de datos PUBMED y SCIELO, sin restricción de período. Se hizo el análisis de los títulos y resúmenes y luego el acceso al texto completo. Resultados: Entre las características del sistema CAD / CAM, los estudios citaron menor necesidad de consultas y tiempo clínico. Se necesita conocimiento de la tecnología por el cirujano-dentista y el mayor costo de laboratorio es una de las desventajas de su utilización. En cuanto a las propiedades, se observa la reducción de la rugosidad y porosidad, así como de la adhesión de Candida albicans a la base de la prótesis y contenido de monómero residual. La mayor dureza de superficie se verifica en comparación con las prótesis totales convencionales. Conclusión: Menor número de consultas y tiempo clínico son características del uso del sistema CAD / CAM en prótesis total. La mayor dureza de la prótesis y reducción de la rugosidad, porosidad, adhesión de C. albicans y del contenido de monómero residual son propiedades que las prótesis totales presentan cuando confeccionadas por esa tecnología. Descriptores: Odontología; Dentadura Completa; Diseño Asistido por Computador.

\section{INTRODUÇÃO}

A tecnologia CAD/CAM possui múltiplas aplicações protéticas, incluindo a fabricação de restaurações intra e extracoronárias, coroas, próteses fixas parciais e, mais recentemente, a construção de próteses totais e maxilofaciais ${ }^{1}$.

A introdução da tecnologia CAD/CAM na fabricação de prótese total (PT) marcou o início de uma nova era na prótese removível ${ }^{2}$. Seu uso para essa produção apresentou crescimento exponencial no mercado odontológico e o número de sistemas CAD/CAM comercialmente disponíveis aumenta a cada ano $^{3}$. Essa tecnologia tem sido amplamente empregada com sucesso na Odontologia ${ }^{4}$.

São vantagens no processo de fabricação de próteses totais por esse sistema a melhor adaptação da prótese e diminuição do número de retornos do paciente ao consultório, o que tem atraído os cirurgiões-dentistas e os próprios pacientes ${ }^{5}$. Ademais, esse processo de fabricação proporciona melhor previsibilidade dos resultados desejados, permitindo alta precisão do ajuste da prótese, graças à eliminação da contração de polimerização, presente 
nas bases convencionais das próteses totais ${ }^{6,7}$.

Aliada a essas características, há diminuição da porosidade da resina acrílica quando comparada à porosidade observada na prótese confeccionada pelo método convencional, o que provoca menor retenção de micro-organismos, em especial de Candida albicans na base da prótese ${ }^{6}$. Tais vantagens se tornam ainda mais importantes no contexto do envelhecimento da população ${ }^{8}$.

CAD/CAM é uma sigla na língua inglesa para Computer-Aided Design e Computer-Aided Manufactoring que significam, respectivamente: desenho auxiliado por computação e manufatura auxiliada por computação. A indústria de maneira geral utiliza esse processo com o objetivo de automatizar, agilizar e controlar os processos de fabricação. O processo CAM, ou a manufatura auxiliada pelo computador, nada mais é do que a materialização ou fabricação da imagem virtual trabalhada no software CAD (desenho realizado através do computador) ${ }^{9,10}$.

A tecnologia CAD/CAM refere-se ao design e fabricação digital. $\mathrm{O}$ software $\mathrm{CAD}$ reconhece a geometria de um objeto enquanto o software CAM é usado para a fabricação ${ }^{11}$. As primeiras tentativas para o desenvolvimento de um sistema assistido por computador para projetar e fabricar próteses removíveis foram realizadas por Maeda et al. ${ }^{12}$, o qual fabricou uma prótese total.

$\mathrm{O}$ processo de fabricação $\mathrm{CAD} / \mathrm{CAM}$ pode incluir a fabricação de aditivos (por prototipagem rápida) ou subtrativa (usinagem de controle numérico de computador-CNC; moagem). A técnica de fabricação subtrativa baseia-se na moagem do produto de um bloco por uma máquina $\mathrm{CNC}$. O software CAM transfere automaticamente o modelo $\mathrm{CAD}$ para a máquina $\mathrm{CNC}^{13}$.

AvaDent e Dentca são dois fabricantes comerciais disponíveis de próteses removíveis totais por CAD/CAM, e utilizam um dispositivo para transferir a relação maxilomandibular para um articulador digital. No processo utilizado por AvaDent, as bases da prótese são fresadas usando uma técnica subtrativa da resina pré-polimerizada. A técnica Dentca usa um processo aditivo, onde uma prótese provisória pode ser preparada, se o dentista requerer, através de prototipagem rápida ${ }^{11}$.

Os modelos podem ser preparados usando impressão convencional ou impressão digital intraoral. Após a impressão, o próximo passo é a transferência da relação maxilomandibular, que pode ser realizada por técnicas convencionais, ou pelo kit dos sistemas comerciais disponíveis ${ }^{11}$.

São necessárias duas consultas clínicas para a fabricação de próteses removíveis totais utilizando os sistemas Avadent e Dentca. No primeiro compromisso, as impressões são gravadas. Então, a relação maxilomandibular é obtida usando dispositivo de medição anatômica. A dimensão vertical de oclusão é determinada usando métodos convencionais. Posteriormente, a relação cêntrica é obtida e os dentes são selecionados. A última etapa do primeiro compromisso é a entrega da impressão final ao fabricante. No segundo compromisso clínico, as próteses são entregues e todos os ajustes oclusais realizados. Essas etapas são semelhantes às da entrega da prótese convencional ${ }^{11}$.

A resina de polimetilmetacrilato ativada por calor (PMMA) é o material mais utilizado para a construção de próteses removíveis ${ }^{14}$ devido às suas características físicas e químicas, facilidade de processamento e custo razoável. No entanto, existem algumas propriedades limitantes, o mais importante é a liberação de monômero residual de metacrilato de metila (MMA) que afeta a estabilidade dimensional, a aderência de bactérias orais, como $C$. albicans à resina e as consultas prolongadas necessárias para a fabricação das próteses.

A presença de monômero residual em resinas das bases da PT afeta as propriedades mecânicas e a biocompatibilidade, e a lixiviação do monômero dá origem a reações alérgicas com sintomas como sensações de queimação, estomatite, edema e ulceração da mucosa oral ${ }^{15}$.

Os sistemas CADICAM oferecem inúmeros benefícios clínicos, uma vez que os discos PMMA utilizados para a fresagem da PT são polimerizados sob alta temperatura e pressão, um processo que promove a formação de cadeias de polímero mais longas que conduzem a um maior grau de conversão de monômeros e valores mais baixos de monômero residual bem como porosidade mínima ${ }^{16}$.

As condições de temperatura e pressão elevada para a polimerização de resinas de CAD/CAM e a adição de agentes inorgânicos restringem a contração de polimerização dimensional e reforçam as propriedades mecânicas dessas resinas, incluindo dureza e resistência ao desgaste ${ }^{17,18}$. Tais propriedades também diminuem a deterioração da superfície e a adesão da placa bacteriana à prótese ${ }^{19}$.

Embora os sistemas CAD/CAM sejam opção comercialmente disponível como técnica alternativa à base convencional de resina acrílica de próteses totais, são sistemas cujas propriedades ainda não foram totalmente investigadas ${ }^{4}$.

Assim, o presente estudo tem como objetivo realizar uma revisão de literatura sobre a aplicabilidade dos sistemas CAD/CAM na fabricação de próteses totais, a fim de elucidar as propriedades das próteses confeccionadas através dessa tecnologia e as características desse sistema.

\section{MATERIAL E MÉTODO}

Realizou-se revisão de literatura baseada na pesquisa de artigos científicos obtidos das bases de dados: PUBMED e SCIELO, com as seguintes 
palavras-chave: Prótese total; CAD/CAM; desenho auxiliado por computação/manufatura auxiliada por computação.

Foram incluídos os estudos que relataram as características dos sistemas CAD/CAM em prótese total, assim como os que investigaram as propriedades das próteses totais fabricadas por essa tecnologia. Não houve restrição de período para a pesquisa. Foram excluídos os artigos científicos que não se enquadraram ao tema proposto e aos critérios de inclusão. O processo de busca foi executado em três fases através da busca de títulos, análise de resumos e identificação de artigos de texto completo.

RESULTADOS E DISCUSSÃO

\begin{tabular}{rllrl}
\hline Dentre & as & vantagens & das & próteses \\
confeccionadas & por & CAD/CAM & se & observa:
\end{tabular}
diminuição do número de consultas, melhor ajuste das próteses dentárias, redução do risco de colonização de micro-organismos nas superfícies da prótese e menor incidência de estomatite protética, avanços na padronização para pesquisa clínica sobre próteses removíveis, reprodução fácil da prótese e controle de qualidade superior por clínicos e técnicos ${ }^{11,20}$.

As desvantagens citadas na literatura são: desafio de fabricação causado pela tomada de impressão e procedimentos de gravação de dimensão vertical de oclusão, transferência de relação maxilomandibular e manutenção do suporte de lábios, que são todos similares aos procedimentos utilizados no processo convencional. Incapacidade de definir o plano oclusal mandibular, materiais caros e aumento do custo de laboratório em comparação com os métodos convencionais ${ }^{11}$.

A resina acrílica da base das próteses por $\mathrm{CAD} / \mathrm{CAM}$ é fornecida como blocos prépolimerizados que são produzidos em condições controladas industrialmente com parâmetros de pressão e temperatura padronizados. Esses blocos, que são facilmente moídos, ganharam popularidade nos últimos anos na fabricação de próteses removíveis. É crucial examinar as propriedades mecânicas, como a resistência à flexão, o módulo de flexão e a dureza da superfície ${ }^{21}$.

Ayman $^{22} 2017$ realizou uma pesquisa onde a força de flexão e a dureza da superfície foram testadas e o teor de monômero residual foi estimado usando cromatografia em fase gasosa e verificou que PMMA ativado por calor, mostrou maior força de flexão e módulo de flexão de baixo valor em comparação com a base da prótese de resina acrílica fabricada por CAD/CAM, nesta foi verificada maior dureza e valores menores de monômero residual.

Em contrapartida, a pesquisa de Steinmassl et $\mathrm{al}^{5} 2017$ demonstrou que todas as próteses testadas confeccionadas por CAD/CAM liberaram quantidades muito baixas de monômero de metacrilato, mas não significativamente menores do que as convencionais termopolimerizadas.

Al-Fouzan et $\mathrm{al}^{20} .2017$ observaram diferença significativa na adesão de $C$. albicans às bases de PT criadas com CAD/CAM e a adesão àqueles criados com o procedimento convencional. As bases das PT por CAD/CAM apresentaram menor adesão de $C$. albicans do que as bases de próteses criadas com o procedimento convencional. Os autores afirmam que a rugosidade de superfície contribui para a colonização de $C$. albicans. Corroborando com essa pesquisa, Alammari ${ }^{4}$ estudou a rugosidade de superfície em resina de poli-metacrilato de metila, resina termoplástica e resina de sistemas CAD/CAM e constatou menor rugosidade superficial nas resinas das bases de próteses confeccionadas por CAD/CAM.

Dessa forma, verifica-se que os sistemas CAD/CAM melhoram as propriedades da resina acrílica presente nas bases das próteses totais, aumentando a dureza de superfície e diminuindo a rugosidade, porosidade, aderência de $C$. albicans e o teor de monômero residual. Para se obter as vantagens que essa tecnologia oferece, isto é, próteses totais de melhor qualidade e em menor tempo clínico, o cirurgião-dentista deve estar capacitado ao manuseio do equipamento.

\section{CONCLUSÃO}

Os sistemas CAD/CAM anunciaram uma nova fase para as próteses removíveis, com sua utilização na confecção de próteses totais. Os estudos revelaram características do uso dessa tecnologia como a diminuição no número de visitas do paciente ao consultório odontológico e tempo reduzido das consultas clínicas. Os ajustes tornaram-se altamente precisos e as próteses confeccionadas por esse sistema apresentam boa retenção. Com a eliminação da contração de polimerização, melhorias nas propriedades da prótese são destacadas como a redução da rugosidade e porosidade, assim como da adesão de $C$. albicans à base da prótese e teor de monômero residual. Maior dureza de superfície é observada em comparação com as próteses totais convencionais.

\section{REFERÊNCIAS}

1. Albero A, Pacual A, Camps I, Grau-Benitez M. Comparative characterization of a novel cad-cam polymer-infiltrated-ceramic-network. J Clin Exp Dent. 2015;7(4):e495-500.

2. Janeva N, Kovacevska G, Janev E. Complete Dentures Fabricated with CAD/CAM Technology and a Traditional Clinical Recording Method. Open Access Macedonian Journal of Medical Sciences. 2017;5(6):785-89.

3. Baba NZ, Alrumaih HS, Goodacre BJ, Goodacre CJ. Current techniques in CAD/CAM denture 
fabrication. Gen Dent. 2016;64(6):23-8.

4. Alamari MR. The influence of polishing techniques on pre-polymerized CAD/CAM acrylic resin denture bases. Electronic Physician. 2017;9(10):5452-58.

5. Steinmassl PA, Klaunzer F, Steinmassi O, Dumfahrt H, Grunert I. Evaluation of Currently Available CAD/CAM Denture Systems. Int $\mathbf{J}$ Prosthodont. 2017;30(2):116-22.

6. de Mendonça AF, Furtado de Mendonça M, White GS, Sara G, Littlefair D. Total CAD/CAM Supported Method for Manufacturing Removable Complete Dentures. Case Reports in Dentistry. 2016; 2016:1259581.

7. Mclaughlin JB, Ramos VJr. Complete denture fabrication with CAD/CAM record bases. J Prosthet Dent. 2015;114(4):493-97.

8. Schweiger J, Guth JF, Edelhoff D, Stumbaum J. Virtual evaluation for CAD-CAM-fabricated complete dentures. J Prosthet Dent. 2017; 117(1):28-33.

9. Liu PR, Essig ME. Panorama of dental CAD/CAM restorative systems. Compend Contin Educ Dent. 2008; 29:482, 4, 6-8 passim.

10. Giordano R. Materials for chairside CAD/CAMproduced restorations. J Am Dent Assoc. 2006; 137(Suppl):14S-21S.

11. Bilgin MS, Baytaroglu EN, Erdem A, Dilber E. A review of computer-aided design/computeraided manufacture techniques for removable denture fabrication. European Journal of Dentistry. 2016;10(2):286-91.

12. Maeda $\mathrm{Y}$, Minoura M, Tsutsumi S, Okada M, Nokubi T. A CAD/CAM system for removable denture. Part I: Fabrication of complete dentures. Int J Prosthodont. 1994;7:17-21.

13. Di Giacomo GA, Cury PR, de Araujo NS, Sendyk WR, Sendyk CL. Clinical application of stereolithographic surgical guides for implant placement: Preliminary results. J Periodontol. 2005; 76:503-7.

14. Ucar Y, Akova T, Aysan I. Mechanical properties of polyamide versus different PMMA denture base materials. J Prosthodont. 2012; 21(3):173-6.

15. Lee HJ, Kim CW, Kim YS. The level of residual monomer in injection molded denture base materials. J Korean Acad Prosth. 2003; 41(3):360-8.

16. Kattadiyil MT, Goodacre CJ, Baba NZ. CAD/CAM complete dentures: a review of two commercial fabrication systems. J Calif Dent Assoc. 2013; 41(6):407-16.

17. Consani RLX, Pucciarelli MGR, Mesquita MF, Nogueira MC, Barao VA. Polymerisation cycles on hardness and surface gloss of denture base. Int J Contemp Dent Med Rev. 2014;2014:1-6.
18. Nakayama M, Takagi N. Methods for manufacturing dental resin block. US: 2016.

19. Murakami N, Wakabayashi N, Matsushima R, Kishida A, Igarashi Y. Effect of high-pressure polymerization on mechanical properties of PMMA denture base resin. J Mech Behav Biomed Mater. 2013;20:98-104.

20. Al-Fouzan AF, Al-Mejarad Lamya A, Albarrag AM. Adherence of Candida to complete denture surfaces in vitro: A comparison of conventional and CAD/CAM complete dentures. J Adv Prosthodont. 2017;9(5):402-8.

21. Digholkar S, Madhav VN, Palaskar J. Evaluation of the flexural strength and microhardness of provisional crown and bridge materials fabricated by different methods. J Indian Prosthodont Soc. 2016;16(4):328-34.

22. Ayman AD. The residual monomer content and mechanical properties of CADICAM resins used in the fabrication of complete dentures as compared to heat cured resins. Electron Physician. 2017;9(7):4766-72.

\section{CONFLITO DE INTERESSES}

Os autores declaram não haver conflitos de interesse.

\section{AUTOR PARA CORRESPONDENCIA}

\section{Julliana Cariry Palhano Freire}

jullianapalhano@hotmail.com

Submetido em 17/04/2018

Aceito em 27/06/2018 